\title{
Modular application of an Integration by Fractional Expansion (IBFE) method to multiloop Feynman diagrams II
}

\author{
Iván González* \\ Departamento de Física \\ Pontificia Universidad Católica de Santiago, \\ Santiago, Chile. \\ Iván Schmidt ${ }^{\dagger}$ \\ Departamento de Física y Centro de Estudios Subatómicos, \\ Universidad Técnica Federico Santa María, \\ Valparaiso, Chile
}

\begin{abstract}
A modular application of the integration by fractional expansion method for evaluating Feynman diagrams is extended to diagrams that contain loop triangle subdiagrams in their geometry. The technique is based in the replacement of this module or subdiagram by its corresponding multiregion expansion (MRE), which in turn is obtained from Schwinger's parametric representation of the diagram. The result is a topological reduction, transforming the triangular loop into an equivalent vertex, which simplifies the search for the MRE of the complete diagram. This procedure has important advantages with respect to considering the parametric representation of the whole diagram: the obtained MRE is reduced and the resulting hypergeometric series tend to have smaller multiplicity.
\end{abstract}

PACS : 11.25.Db; 12.38.Bx

Keywords : Perturbation theory; Scalar integrals; Multiloop Feynman diagrams; Schwinger parameters; Negative Dimension Integration Method (NDIM), Integration by Fractional Expansion (IBFE).

*e-mail: igonzalez@fis.puc.cl

†e-mail: ivan.schmidt@usm.cl 


\section{Introduction}

The integration by fractional expansion technique (IBFE) constitutes a rather simple method that allows to evaluate Feynman diagrams starting from the corresponding Schwinger's parametric representation, and whose main advantage is to transfer the complexity of the direct calculation of multidimensional integrals to the evaluation of linear systems of equations that the method generates. This technique is particularly useful when the propagator exponents are arbitrary, and the general solutions obtained are sums of generalized hypergeometric functions, whose number of variables depends on the number of invariants in the diagram and also on the topological family to which the diagram belongs.

As is the case with any integration technique, although IBFE can be applied to any diagram, it is not always possible to obtain simple solutions due to the number and multivariability of the resulting hypergeometric series. Nevertheless, for certain families of diagrams its application is particularly simple and optimal. In a previous work [2] we showed that a modular application of IBFE is a tool that simplifies considerably the complexity of the solutions: specifically we considered bubble-type modules or subgraphs. Here we extend this modular application to cases in which the modules are triangular loops, which then provides a rather simple procedure to find the solutions to a large number of Feynman diagrams.

This work is divided in the following way. In Sec. II we apply the IBFE technique to the massless triangular module, and find its representing multiregion expansion for the case of off-shell external lines. Here we define the one-loop function $G_{T}$, which allows to describe the multiregion expansion (MRE) of this module as the sum over this loop function and a vertex, thus effectively reducing the loop to a node. One of the main characteristic features of the Feynman diagram general solutions, when the propagators have arbitrary powers, is that they are given by sums of multivariable hypergeometric functions. The arguments or variables in these functions are ratios between invariants associated to the graph, and given the structure of the resulting hypergeometric function, the convergence condition requires that at least this ratio of invariants be less than unity. Therefore from a particular diagram MRE different solutions can be extracted, each of them associated to a particular kinematical regime, where the differentiating element is determined by the ratio of two invariants. This form of differentiating the solutions is direct for certain families of diagrams 2. Once all terms associated to a specific region of interest are found, summing them will give us the desired solution. Nevertheless, for topologies that do not belong to the previously mentioned family, and which is the most general case, this criteria for finding the correct terms of the solutions extracted from the MRE actually fails, because in the general case the application of IBFE can generate solutions which contain hypergeometric functions with a particular argument, the unity. Given the structure of the diagram solutions, this value corresponds to the ratio of two invariants $(A / B)$ or $(B / A)$, when $A=B$. Nevertheless, both ratios are associated to different regions (different solutions) and the fact that they are equal does not allow to differentiate them. Here we present a method that solves this problem, and apply it to the specific case of diagrams that contain triangle subdiagrams.

In Sec. III we evaluate two different two-loop diagrams which contain the triangle subgraph in their topology. The first corresponds to a correction to a three-point function, with six propagators. We find the general solution for arbitrary propagator powers, and then particularize it to unity. In order to validate this technique, we compare the solution with the one obtained from a conventional method IBP (integration by parts) 4 . The second diagram is a self-energy correction with five propagators, for which we present a new solution for the case or arbitrary values of the propagator powers. Finally in Sec. $I V$ we describe some cases in which this massless triangle function can be used and the generalization to massive loops.

\section{Triangular module}

In this section we will find the MRE associated to the one-loop triangular subgraph or module, considering a case in which the propagators are massless and the external lines are off-shell. From the MRE of this graph we will define the one-loop function $G_{T}$, which will allow to represent this module as a particular sum of three line vertices. This formulation will then be applied as examples to a self-energy and a three-point function diagram, both with two loops. The self-energy example will show quite clearly the advantages of this modular procedure. 


\subsection{Obtaining the MRE of the triangular module}

We start from the momentum integral representation for this graph, which is given in the massless case and in $D$ dimensions by:

$$
G=\int \frac{d^{D} q}{i \pi^{D / 2}} \frac{1}{\left(\left(p_{1}+q\right)^{2}\right)^{a_{1}}\left(\left(p_{1}+p_{2}+q\right)^{2}\right)^{a_{2}}\left(q^{2}\right)^{a_{3}}} .
$$

When the propagator powers $\left\{a_{i}\right\}$ are integers, this expression can be easily reduced to simpler topologies using the integration by parts technique [4]. The situation is more difficult for arbitrary indices $\left\{a_{i}\right\}$, which we are considering in this work. Graphically the integral can be represented by the following diagram:

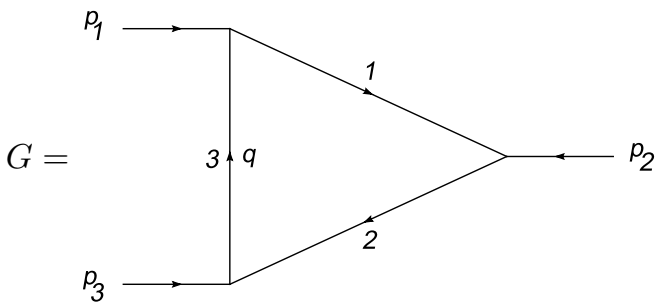

The IBFE integration technique is applied to Schwinger's parametric representation of the diagram, which can be obtained from the matrix of parameters [3] associated to Eq. (11). In the present case this matrix can be easily obtained and it is given by:

$$
\mathbf{M}=\left(\begin{array}{ccc}
x_{1}+x_{2}+x_{3} & x_{1}+x_{2} & x_{2} \\
x_{1}+x_{2} & x_{1}+x_{2} & x_{2} \\
x_{2} & x_{2} & x_{2}
\end{array}\right),
$$

which allows to express Schwinger's parametric representation as:

$$
G=\frac{(-1)^{-D / 2}}{\Gamma\left(a_{1}\right) \Gamma\left(a_{2}\right) \Gamma\left(a_{3}\right)} \int_{0}^{\infty} d \vec{x} \frac{\exp \left(-\frac{C_{11} p_{1}^{2}+2 C_{12} p_{1} \cdot p_{2}+C_{22} p_{2}^{2}}{U}\right)}{U^{D / 2}},
$$

where $d \vec{x}=x_{1}^{a_{1}-1} x_{2}^{a_{2}-1} x_{3}^{a_{3}-1} d x_{1} d x_{2} d x_{3}$ and $U=\left(x_{1}+x_{2}+x_{3}\right)$. The coefficients $C_{i j}$ can be obtained from the determinants of the submatrices of the matrix of parameters (3):

$$
\begin{aligned}
& C_{11}=\left|\begin{array}{cc}
x_{1}+x_{2}+x_{3} & x_{1}+x_{2} \\
x_{1}+x_{2} & x_{1}+x_{2}
\end{array}\right|=x_{3}\left(x_{1}+x_{2}\right), \\
& C_{12}=\left|\begin{array}{cc}
x_{1}+x_{2}+x_{3} & x_{2} \\
x_{1}+x_{2} & x_{2}
\end{array}\right|=x_{2} x_{3}, \\
& C_{22}=\left|\begin{array}{cc}
x_{1}+x_{2}+x_{3} & x_{2} \\
x_{2} & x_{2}
\end{array}\right|=x_{2}\left(x_{1}+x_{3}\right) .
\end{aligned}
$$

Replacing in (44) and remembering that $p_{3}^{2}=\left(p_{1}+p_{2}\right)^{2}=p_{1}^{2}+2 p_{1} \cdot p_{2}+p_{2}^{2}$, we get after a little algebra Schwinger's parametric representation of (1):

$$
G=\frac{(-1)^{-D / 2}}{\prod_{j=1}^{3} \Gamma\left(a_{j}\right)} \int_{0}^{\infty} d \vec{x} \frac{\exp \left(-\frac{x_{3} x_{1}}{U} p_{1}^{2}\right) \exp \left(-\frac{x_{1} x_{2}}{U} p_{2}^{2}\right) \exp \left(-\frac{x_{2} x_{3}}{U} p_{3}^{2}\right)}{U^{D / 2}} .
$$

Now we will deduce the MRE of the parametric integral (6). For this purpose we expand the exponentials that are present in the integrand, which leads us to the following multiple series: 


$$
G=\frac{(-1)^{-D / 2}}{\prod_{j=1}^{3} \Gamma\left(a_{j}\right)} \sum_{n_{1}, . ., n_{3}} \phi_{n_{1}, . ., n_{3}}\left(p_{1}^{2}\right)^{n_{1}}\left(p_{2}^{2}\right)^{n_{2}}\left(p_{3}^{2}\right)^{n_{3}} \int_{0}^{\infty} d \vec{x} \frac{x_{1}^{n_{1}+n_{2}} x_{2}^{n_{2}+n_{3}} x_{3}^{n_{1}+n_{3}}}{U^{\frac{D}{2}+n_{1}+n_{2}+n_{3}}},
$$

and this allows us to obtain the MRE of the polynomial $U=\left(x_{1}+x_{2}+x_{3}\right)$. For the denominator in the integral we have that:

$$
\frac{1}{\left(x_{1}+x_{2}+x_{3}\right)^{\frac{D}{2}+n_{1}+n_{2}+n_{3}}}=\sum_{n_{4}, . ., n_{6}} \phi_{n_{4}, . ., n_{6}} x_{1}^{n_{4}} x_{2}^{n_{5}} x_{3}^{n_{6}} \frac{\left\langle\frac{D}{2}+n_{1}+n_{2}+n_{3}+n_{4}+n_{5}+n_{6}\right\rangle}{\Gamma\left(\frac{D}{2}+n_{1}+n_{2}+n_{3}\right)},
$$

and then, integrals already separated in factors of the form $\int d x x^{\alpha+\ldots-1}$, can be replaced by the equivalent constraints $\langle\alpha+\ldots\rangle$. This is the final step of the fractional expansion process, and we have finally obtained the MRE of the diagram from its parametric representation (6):

$$
G=\frac{(-1)^{-D / 2}}{\prod_{j=1}^{3} \Gamma\left(a_{j}\right)} \sum_{n_{1}, . ., n_{6}} \phi_{n_{1}, . ., n_{6}}\left(p_{1}^{2}\right)^{n_{1}}\left(p_{2}^{2}\right)^{n_{2}}\left(p_{3}^{2}\right)^{n_{3}} \frac{\prod_{j=1}^{4} \Delta_{j}}{\Gamma\left(\frac{D}{2}+n_{1}+n_{2}+n_{3}\right)}
$$

Where we have summarized the constraints $\left\{\Delta_{j}\right\}$ in the following identities:

$$
\left\{\begin{aligned}
\Delta_{1} & =\left\langle\frac{D}{2}+n_{1}+n_{2}+n_{3}+n_{4}+n_{5}+n_{6}\right\rangle, \\
\Delta_{2} & =\left\langle a_{1}+n_{1}+n_{2}+n_{4}\right\rangle \\
\Delta_{3} & =\left\langle a_{2}+n_{2}+n_{3}+n_{5}\right\rangle \\
\Delta_{4} & =\left\langle a_{3}+n_{1}+n_{3}+n_{6}\right\rangle .
\end{aligned}\right.
$$

From expression (9) we see that the composition of sums and Kronecker deltas $\left(\approx \Delta_{k}\right)$ of the MRE of this module or graph is $6 \Sigma / 4 \delta$.

\subsection{Definition of the one-loop function $G_{T}$}

In order to generate a systematic reduction procedure of interior triangle subgraphs of a more complex topology, we define from Eq. (9) a loop function denoted $G_{T}$, such that (9) can be written as:

$$
G=\sum_{n_{1}, . ., n_{3}} G_{T}\left(a_{1}, a_{2}, a_{3} ; n_{1}, n_{2}, n_{3}\right) \frac{1}{\left(p_{1}^{2}\right)^{-n_{1}}} \frac{1}{\left(p_{2}^{2}\right)^{-n_{2}}} \frac{1}{\left(p_{3}^{2}\right)^{-n_{3}}}
$$

where the one-loop function $G_{T}\left(a_{1}, a_{2}, a_{3} ; n_{1}, n_{2}, n_{3}\right)$ by comparison is given by the expression:

$$
G_{T}\left(a_{1}, a_{2}, a_{3} ; n_{1}, n_{2}, n_{3}\right)=\frac{(-1)^{-D / 2}}{\prod_{j=1}^{3} \Gamma\left(a_{j}\right)} \sum_{n_{4}, . ., n_{6}} \phi_{n_{1}, . ., n_{6}} \frac{\prod_{j=1}^{4} \Delta_{j}}{\Gamma\left(\frac{D}{2}+n_{1}+n_{2}+n_{3}\right)}
$$

Let us look at the propagator structure of the Eq. (11). It is clear that it can be represented pictorially as a sum whose argument contains the original diagram with the loop reduced to an effective vertex where the external lines reach, as inverse propagators. The cost of this topological minimization is a multiple sum. Graphically the representation of the MRE of the triangular module is given by:

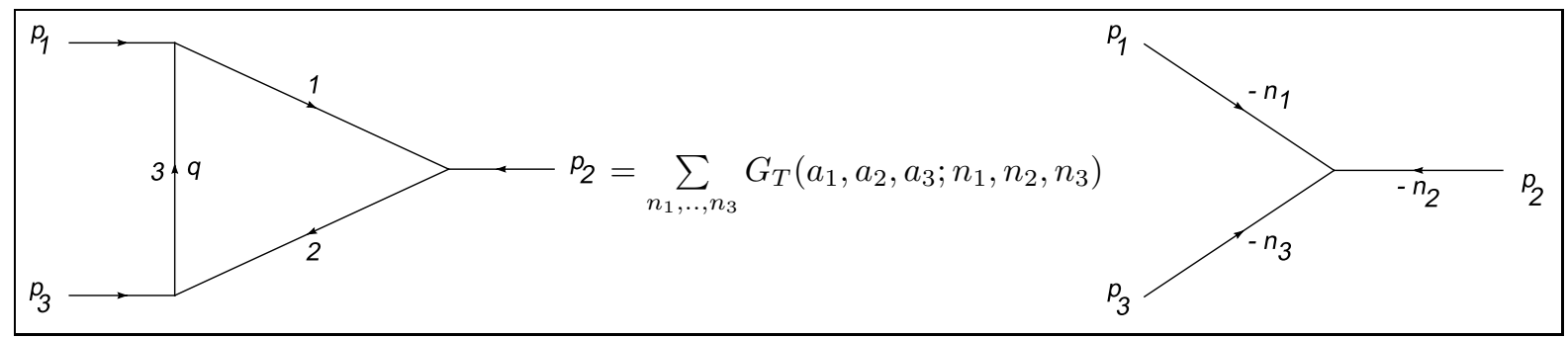


Therefore we have shown that it is possible to reduce topologies which contain this type of vertices, just as was done with the reduction of propagator bubbles [1].

\subsection{Method for differentiating series of unitary argument}

Before going into the actual application of IBFE to diagrams with triangle subgraphs, it is necessary to implement a method that allows to discriminate whether a unitary argument in the series that comes from the MRE, corresponds to the limit of the ratio of invariants in a particular region or to its complementary region. This is based on multiplying the external momenta by certain constants (fictitious invariants) $A_{i}(i=1,2,3)$. Once the solution to the diagram is found, the arguments of the obtained hypergeometric series correspond now to ratios of these constants, allowing to separate the solutions associated to different regions of the hypergeometric functions whose arguments are $\left(\frac{A_{i}}{A_{k}}\right)$ and $\left(\frac{A_{k}}{A_{i}}\right)$, with $i \neq k$. Once these structures have become separated, we take $A_{1}=A_{2}=A_{3}=1$, and the correct solution is obtained. This simple method solves the problem of summing hypergeometric series, with the same argument, that actually belong to different solutions of the original problem. In the triangle case, we transform the external momenta in (9) as follows:

$$
p_{i}^{2} \Longrightarrow A_{i} p_{i}^{2} .
$$

This transformation allows to obtain the modified expression of (13):

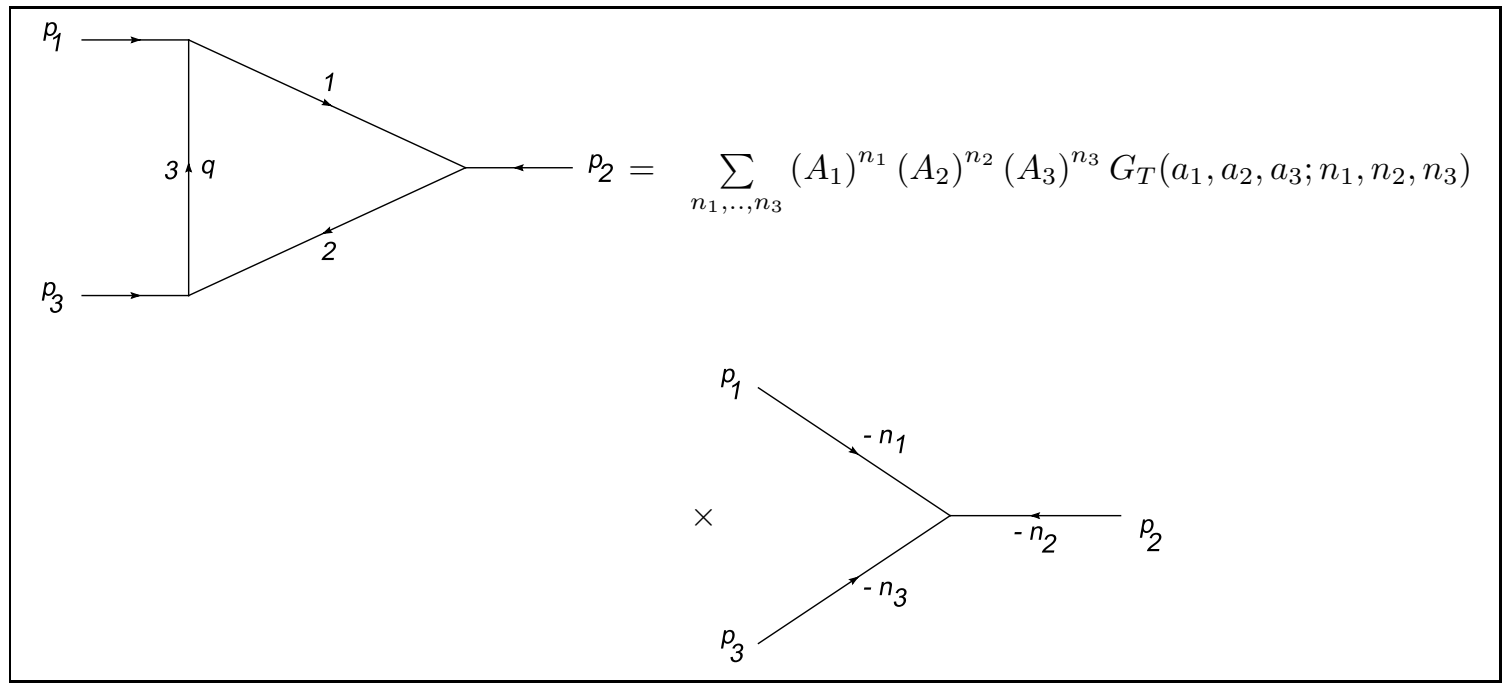

\section{Applications}

The topological reduction formula (15), which corresponds to an MRE of the triangular module, will be useful for finding the MRE of generic graphs that contain in their geometry one or more of these triangular modules. We will now explain how to use formula (15) in specific cases, showing the advantages of this procedure.

\subsection{Example I : Two-loop triangle}

As the first example of the modular reduction technique and of the topological formula (15), let us consider a diagram $g$ with three external lines, two loops and six propagators. We take a massless theory, with mass shell external lines $K_{1}$ and $K_{2}\left(K_{1}^{2}=K_{2}^{2}=0\right)$. The diagram is: 


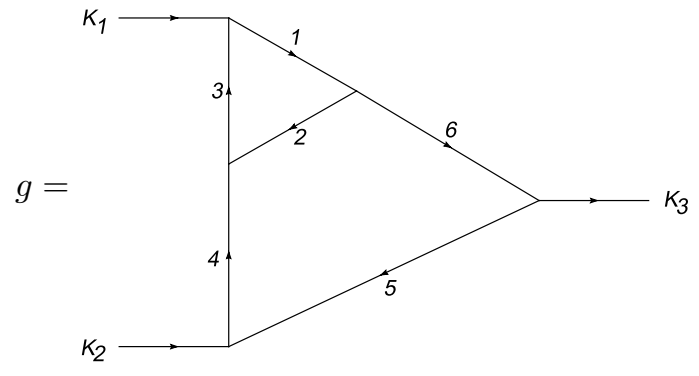

\subsubsection{Getting the diagram MRE}

The first step in order to find the diagram MRE with the modular procedure is to reduce the subgraph associated to the indices $\left\{a_{1}, a_{2}, a_{3}\right\}$, using formula (15). In this way we obtain an expression where we have eliminated one of the loops of the diagram, which has been replaced by an effective vertex where the three propagators associated to the indices $\left\{-n_{1},-n_{2},-n_{3}\right\}$ meet. In detail:

$$
g=\sum_{n_{1}, \ldots, n_{3}}\left(A_{1}\right)^{n_{1}}\left(A_{2}\right)^{n_{2}}\left(A_{3}\right)^{n_{3}} G_{T}\left(a_{1}, a_{2}, a_{3} ; n_{1}, n_{2}, n_{3}\right)
$$

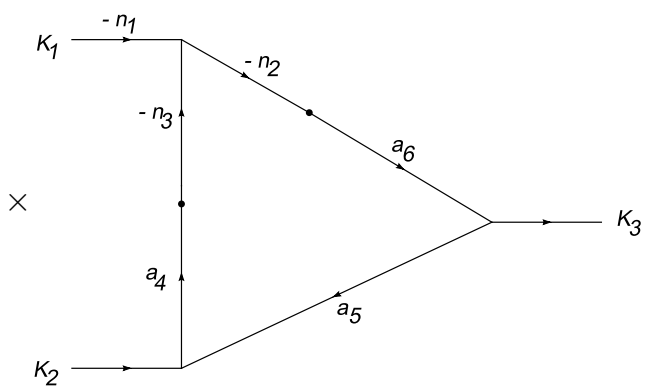

In Eq. (17) we can see that one of the external lines, the one associated with the momentum $K_{1}$, is affected by the modular reduction on $g$. It is possible to actually extract this propagator $\Longrightarrow \frac{1}{\left(K_{1}^{2}\right)^{-n_{1}}}$ from the diagram, since it is not involved in the loop integration, and write it as a factor that multiplies this graph. Looking at Eq. (11), and its pictorial equivalent (13), we conclude that it is possible to eliminate from the graph the information related to the index of summation $n_{1}$. Rewriting (17) we thus have:

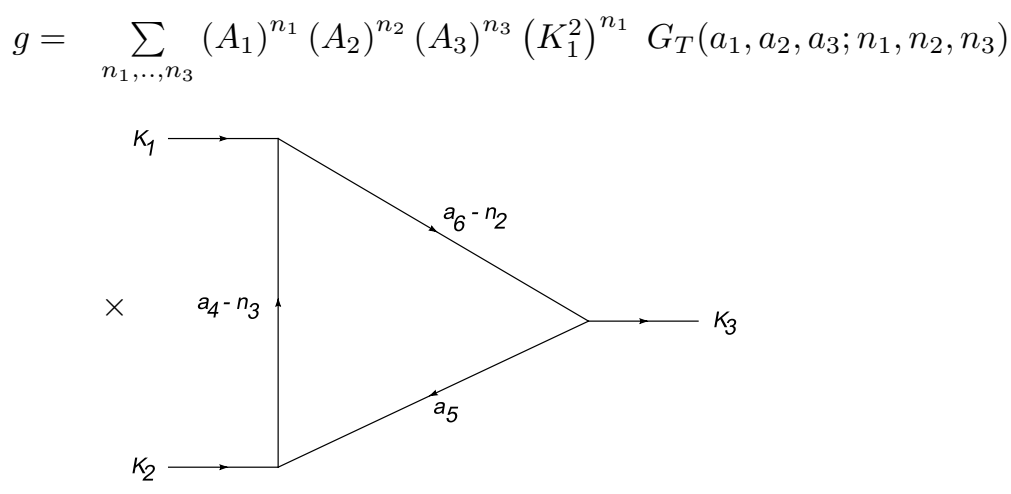

The resulting triangle allows us to apply the reduction formula (13). We then get the MRE of the topology $g$ as: 


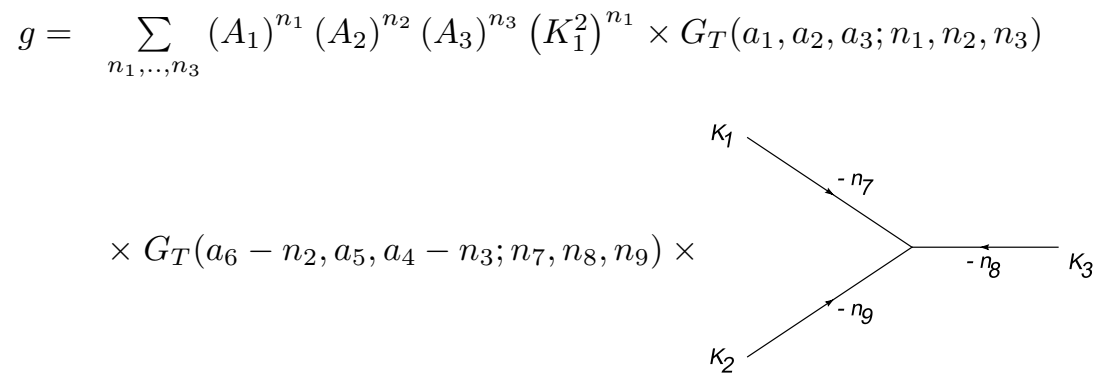

Since each function $G_{T}$ is associated to six summation indices, in order to use the same notation for these indices $\{n\}$, the second function $G_{T}$ starts with the summation index $n_{7}$. Therefore we get:

$$
\begin{aligned}
g= & \sum_{n_{1}, . ., n_{3}}\left(A_{1}\right)^{n_{1}}\left(A_{2}\right)^{n_{2}}\left(A_{3}\right)^{n_{3}}\left(K_{1}^{2}\right)^{n_{1}+n_{7}}\left(K_{2}^{2}\right)^{n_{9}}\left(K_{3}^{2}\right)^{n_{8}} \\
& G_{T}\left(a_{1}, a_{2}, a_{3} ; n_{1}, n_{2}, n_{3}\right) \times G_{T}\left(a_{6}-n_{2}, a_{5}, a_{4}-n_{3} ; n_{7}, n_{8}, n_{9}\right),
\end{aligned}
$$

where after replacing the functions $G_{T}$ by their respective MRE, we obtain the expression:

$$
\begin{aligned}
g= & \digamma \sum_{\{n\}} \phi_{n_{1}, . ., n_{12}}\left(A_{1}\right)^{n_{1}}\left(A_{2}\right)^{n_{2}}\left(A_{3}\right)^{n_{3}}\left(K_{1}^{2}\right)^{n_{1}+n_{7}}\left(K_{2}^{2}\right)^{n_{9}}\left(K_{3}^{2}\right)^{n_{8}} \\
& \frac{\prod_{j=1}^{8} \Delta_{j}}{\Gamma\left(\frac{D}{2}+n_{1}+n_{2}+n_{3}\right) \Gamma\left(\frac{D}{2}+n_{7}+n_{8}+n_{9}\right) \Gamma\left(a_{6}-n_{2}\right) \Gamma\left(a_{4}-n_{3}\right)} .
\end{aligned}
$$

We have defined the factor:

$$
\digamma=\frac{(-1)^{-D}}{\Gamma\left(a_{1}\right) \Gamma\left(a_{2}\right) \Gamma\left(a_{3}\right) \Gamma\left(a_{5}\right)}
$$

The constraints $\left\{\Delta_{j}\right\}$ are given by the following identities:

$$
\left\{\begin{array}{rlrl}
\Delta_{1} & =\left\langle\frac{D}{2}+n_{1}+n_{2}+n_{3}+n_{4}+n_{5}+n_{6}\right\rangle, & & \Delta_{5}=\left\langle\frac{D}{2}+n_{7}+n_{8}+n_{9}+n_{10}+n_{11}+n_{12}\right\rangle, \\
\Delta_{2}=\left\langle a_{1}+n_{1}+n_{2}+n_{4}\right\rangle, & \Delta_{6}=\left\langle a_{4}-n_{3}+n_{7}+n_{8}+n_{10}\right\rangle, \\
\Delta_{3}=\left\langle a_{2}+n_{2}+n_{3}+n_{5}\right\rangle, & \Delta_{7}=\left\langle a_{5}+n_{8}+n_{9}+n_{11}\right\rangle, \\
\Delta_{4}=\left\langle a_{3}+n_{1}+n_{3}+n_{6}\right\rangle, & \Delta_{6}=\left\langle a_{6}-n_{2}+n_{7}+n_{9}+n_{12}\right\rangle .
\end{array}\right.
$$

Finally, applying the conditions of the problem $\left(K_{1}^{2}=K_{2}^{2}=0\right)$, the summation indices $n_{1}, n_{7}$ and $n_{9}$ must vanish in order to have a nonzero solution. Replacing these values and eliminating the sums in (21), we have obtain the MRE of the diagram.

After renaming the indexes $\{n\} \Longrightarrow\{l\}$ so that they become consecutive, the multiregion expansion is finally given by:

$$
\begin{aligned}
g= & \digamma \sum_{\{l\}} \phi_{l_{1}, . ., l_{9}}\left(A_{2}\right)^{l_{1}}\left(A_{3}\right)^{l_{2}}\left(K_{3}^{2}\right)^{l_{6}} \\
& \frac{\prod_{j=1}^{8} \Delta_{j}}{\Gamma\left(\frac{D}{2}+l_{1}+l_{2}\right) \Gamma\left(\frac{D}{2}+l_{6}\right) \Gamma\left(a_{6}-l_{1}\right) \Gamma\left(a_{4}-l_{2}\right)},
\end{aligned}
$$

and now the constraints are given by:

$$
\left\{\begin{aligned}
\Delta_{1} & =\left\langle\frac{D}{2}+l_{1}+l_{2}+l_{3}+l_{4}+l_{5}\right\rangle, & & \Delta_{5}=\left\langle\frac{D}{2}+l_{6}+l_{7}+l_{8}+l_{9}\right\rangle, \\
\Delta_{2} & =\left\langle a_{1}+l_{1}+l_{3}\right\rangle, & & \Delta_{6}=\left\langle a_{4}-l_{2}+l_{6}+l_{7}\right\rangle, \\
\Delta_{3} & =\left\langle a_{2}+l_{1}+l_{2}+l_{4}\right\rangle, & \Delta_{7} & =\left\langle a_{5}+l_{6}+l_{8}\right\rangle, \\
\Delta_{4} & =\left\langle a_{3}+l_{2}+l_{5}\right\rangle, & \Delta_{6} & =\left\langle a_{6}-l_{1}+l_{9}\right\rangle .
\end{aligned}\right.
$$


Therefore the multiplicity of the hypergeometric series that can be extracted from the MRE is one $\Longrightarrow$ hypergeometric series of the type ${ }_{q} F_{(q-1)}$ and at most $C_{8}^{9}=9$ series of this type whose argument for this case is one.

\subsubsection{General IBFE solution}

Now we proceed to find the general solutions associated with the diagram (16) coming from the MRE (24) which represents it. We maintain all the indexes $\left\{a_{j}\right\}$ with arbitrary values, and for notational simplicity make $A_{2}=A$ and $A_{3}=B$.

The solutions we present here correspond to two regions that can be differentiated according to the ratio of the fictitious invariants used in order to separate them: the region where $A>B$ and the region where $B>A$. Both are solutions of $g$, related by analytical continuation. In particular, in the limit $A=B=1$ they become identical, as will be seen later on.

Analytical solution in the region $\left|\frac{A}{B}\right| \leq 1$ Let us first define the following simplifying notation for the indexes $\left\{a_{j}\right\}$ :

$$
a_{i j k \ldots}=a_{i}+a_{j}+a_{k}+\ldots
$$

Moreover, since in practice each constraint $\langle\ldots\rangle$ eliminates a sum in the MRE, the remaining or free sum at the end of the elimination process generates the corresponding hypergeometric representation ${ }_{q} F_{q-1}$ which we will identify as $G_{j}$, since it is the contribution obtained when the summation index $n_{j}$ is free in the multiregion representation of diagram $g$.

With these definitions we can write the solutions for this region as:

$$
g=g\left(\frac{A}{B}\right)=G_{1}+G_{5},
$$

where the terms inside the sum correspond to the following functions:

$$
\begin{aligned}
G_{1}= & (-1)^{-D}\left(K_{3}^{2}\right)^{D-a_{123456}} \frac{\Gamma\left(a_{123}-\frac{D}{2}\right) \Gamma\left(a_{123456}-D\right) \Gamma\left(\frac{D}{2}-a_{13}\right) \Gamma\left(\frac{D}{2}-a_{12}\right) \Gamma\left(\frac{D}{2}-a_{56}\right) \Gamma\left(D-a_{12346}\right)}{\Gamma\left(a_{2}\right) \Gamma\left(a_{3}\right) \Gamma\left(a_{5}\right) \Gamma\left(D-a_{123}\right) \Gamma\left(a_{1234}-\frac{D}{2}\right) \Gamma\left(\frac{3 D}{2}-a_{123456}\right)} \\
& \times B^{\frac{D}{2}-a_{123}}{ }_{3} F_{2}\left(\begin{array}{cc|c}
a_{123}-\frac{D}{2}, & a_{1}, \quad \frac{D}{2}-a_{56} & \frac{A}{B} \\
a_{1234}-\frac{D}{2}, & 1+a_{12}-\frac{D}{2} & B
\end{array}\right),
\end{aligned}
$$

and:

$$
\begin{aligned}
& G_{5}=(-1)^{-D}\left(K_{3}^{2}\right)^{D-a_{123456}} \frac{\Gamma\left(a_{12}-\frac{D}{2}\right) \Gamma\left(\frac{D}{2}-a_{2}\right) \Gamma\left(\frac{D}{2}-a_{13}\right) \Gamma\left(D-a_{12346}\right) \Gamma\left(a_{123456}-D\right) \Gamma\left(D-a_{1256}\right)}{\Gamma\left(a_{1}\right) \Gamma\left(a_{2}\right) \Gamma\left(a_{5}\right) \Gamma\left(D-a_{123}\right) \Gamma\left(a_{34}\right) \Gamma\left(\frac{3 D}{2}-a_{123456}\right)}
\end{aligned}
$$

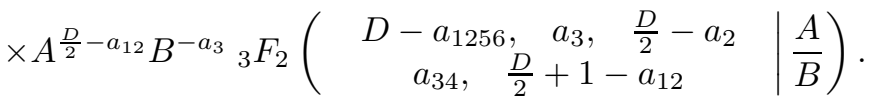

Analytical solution in the region $\left|\frac{B}{A}\right| \leq 1 \quad$ Analogously we have that in this fictitious region the solution is given by:

$$
g=g\left(\frac{B}{A}\right)=G_{2}+G_{3}+G_{7},
$$

with: 


$$
\begin{aligned}
& G_{2}=(-1)^{-D}\left(K_{3}^{2}\right)^{D-a_{123456}} \frac{\Gamma\left(a_{123}-\frac{D}{2}\right) \Gamma\left(\frac{D}{2}-a_{23}\right) \Gamma\left(\frac{D}{2}-a_{13}\right) \Gamma\left(a_{123456}-D\right) \Gamma\left(D-a_{12356}\right) \Gamma\left(D-a_{12346}\right)}{\Gamma\left(a_{1}\right) \Gamma\left(a_{2}\right) \Gamma\left(a_{5}\right) \Gamma\left(a_{4}\right) \Gamma\left(D-a_{123}\right) \Gamma\left(\frac{3 D}{2}-a_{123456}\right)} \\
& \times A^{\frac{D}{2}-a_{123}}{ }_{3} F_{2}\left(\begin{array}{rl|l}
a_{123}-\frac{D}{2}, & 1-a_{4}, \quad a_{3} & \frac{B}{A} \\
1-\frac{D}{2}+a_{23}, & 1-\frac{D}{2}+a_{12356}
\end{array}\right) \\
& G_{3}=(-1)^{-D}\left(K_{3}^{2}\right)^{D-a_{123456}} \frac{\Gamma\left(a_{23}-\frac{D}{2}\right) \Gamma\left(\frac{D}{2}-a_{13}\right) \Gamma\left(\frac{D}{2}-a_{2}\right) \Gamma\left(a_{123456}-D\right) \Gamma\left(\frac{D}{2}-a_{156}\right) \Gamma\left(D-a_{12346}\right)}{\Gamma\left(a_{2}\right) \Gamma\left(a_{3}\right) \Gamma\left(a_{5}\right) \Gamma\left(D-a_{123}\right) \Gamma\left(a_{234}-D\right) \Gamma\left(\frac{3 D}{2}-a_{123456}\right)}
\end{aligned}
$$

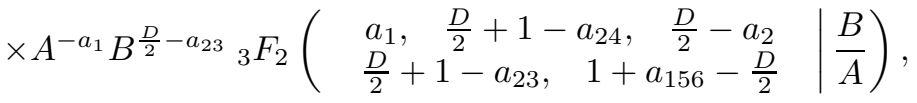

and finally:

$$
\begin{aligned}
G_{7}= & (-1)^{-D}\left(K_{3}^{2}\right)^{D-a_{123456}} \frac{\Gamma\left(a_{23}-\frac{D}{2}\right) \Gamma\left(\frac{D}{2}-a_{13}\right) \Gamma\left(\frac{D}{2}-a_{2}\right) \Gamma\left(a_{123456}-D\right) \Gamma\left(\frac{D}{2}-a_{156}\right) \Gamma\left(D-a_{12346}\right)}{\Gamma\left(a_{2}\right) \Gamma\left(a_{3}\right) \Gamma\left(a_{5}\right) \Gamma\left(D-a_{123}\right) \Gamma\left(a_{234}-D\right) \Gamma\left(\frac{3 D}{2}-a_{123456}\right)} \\
& \times A^{a_{56}-\frac{D}{2}} B^{D-a_{12356}}{ }_{3} F_{2}\left(\begin{array}{c}
D+1-a_{123456}, \quad \frac{D}{2}-a_{56}, \quad D-a_{1256} \\
D+1-a_{12356}, \quad \frac{D}{2}+1-a_{156}
\end{array} \mid \frac{B}{A}\right) .
\end{aligned}
$$

\subsubsection{Particular case : unit indexes}

In the actual Feynman diagram evaluation, in general the corresponding momentum integral has indexes or propagator powers that are equal to unity. From the previous results, making $a_{j}=1(j=1, \ldots, 6)$, we obtain the solution:

Analytic solution in the region $\left|\frac{A}{B}\right| \leq 1$ The solutions in this region are given by:

$$
g=g\left(\frac{A}{B}\right)=G_{1}+G_{5},
$$

where now:

$$
G_{1}=(-1)^{-D}\left(K_{3}^{2}\right)^{D-6} \frac{\Gamma\left(3-\frac{D}{2}\right) \Gamma(6-D) \Gamma\left(\frac{D}{2}-2\right)^{3} \Gamma(D-5)}{\Gamma(D-3) \Gamma\left(4-\frac{D}{2}\right) \Gamma\left(\frac{3 D}{2}-6\right)} \times B^{\frac{D}{2}-3}{ }_{2} F_{1}\left(\begin{array}{c}
1, \quad \frac{D}{2}-2 \\
4-\frac{D}{2}
\end{array} \mid \frac{A}{B}\right),
$$

and:

$$
\begin{aligned}
G_{5}= & (-1)^{-D}\left(K_{3}^{2}\right)^{D-6} \frac{\Gamma\left(2-\frac{D}{2}\right) \Gamma\left(\frac{D}{2}-1\right) \Gamma\left(\frac{D}{2}-2\right) \Gamma(D-5) \Gamma(6-D) \Gamma(D-4)}{\Gamma(D-3) \Gamma\left(\frac{3 D}{2}-6\right)} \\
& \times A^{\frac{D}{2}-2} B^{-1}{ }_{2} F_{1}\left(\begin{array}{cc|c}
D-4, & 1 \\
2 & \frac{A}{B}
\end{array}\right) .
\end{aligned}
$$


Analytical solution in the region $\left|\frac{B}{A}\right| \leq 1$ In this region the solution is:

$$
g=g\left(\frac{B}{A}\right)=G_{2}+G_{3}+G_{7},
$$

where the hypergeometric representations $G_{2,3,7}$ are now given by:

$$
G_{2}=(-1)^{-D}\left(K_{3}^{2}\right)^{D-6} \frac{\Gamma\left(3-\frac{D}{2}\right) \Gamma\left(\frac{D}{2}-2\right)^{2} \Gamma(6-D) \Gamma(D-5)^{2}}{\Gamma(D-3) \Gamma\left(\frac{3 D}{2}-6\right)} \times A_{2}^{\frac{D}{2}-3}{ }_{2} F_{1}\left(\begin{array}{cc|c}
0, & 1 \\
6-D & \frac{B}{A}
\end{array}\right),
$$

or equivalently:

$$
\begin{aligned}
& G_{2}=(-1)^{-D}\left(K_{3}^{2}\right)^{D-6} \frac{\Gamma\left(3-\frac{D}{2}\right) \Gamma\left(\frac{D}{2}-2\right)^{2} \Gamma(6-D) \Gamma(D-5)^{2}}{\Gamma(D-3) \Gamma\left(\frac{3 D}{2}-6\right)} \times A^{\frac{D}{2}-3}, \\
& G_{3}=(-1)^{-D}\left(K_{3}^{2}\right)^{D-6} \frac{\Gamma\left(2-\frac{D}{2}\right) \Gamma\left(\frac{D}{2}-2\right) \Gamma\left(\frac{D}{2}-1\right) \Gamma(6-D) \Gamma(D-5) \Gamma\left(\frac{D}{2}-3\right)}{\Gamma(D-3) \Gamma\left(\frac{3 D}{2}-6\right) \Gamma\left(3-\frac{D}{2}\right)} \\
& \times A^{-1} B^{\frac{D}{2}-2}{ }_{2} F_{1}\left(\begin{array}{c|c}
1, & \frac{D}{2}-2 \\
4-\frac{D}{2} & \frac{B}{A}
\end{array}\right),
\end{aligned}
$$

and finally:

$$
\begin{aligned}
G_{7}= & (-1)^{-D}\left(K_{3}^{2}\right)^{D-6} \frac{\Gamma\left(2-\frac{D}{2}\right) \Gamma\left(\frac{D}{2}-2\right) \Gamma\left(\frac{D}{2}-1\right) \Gamma(6-D) \Gamma\left(\frac{D}{2}-3\right) \Gamma(D-5)}{\Gamma(D-3) \Gamma\left(\frac{3 D}{2}-6\right)} \\
& \times A^{2-\frac{D}{2}} B^{D-5}{ }_{1} F_{0}\left(\begin{array}{c}
D-5 \\
-
\end{array}\right.
\end{aligned}
$$

\subsubsection{Analytic continuation and dependence between the solutions obtained with IBFE}

It is possible to show that in the limit $A=B=1$ the solutions (34) and (37) are equal, and therefore both are related by analytic continuation. For this purpose we use the following identity:

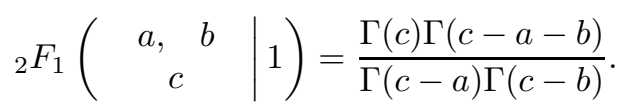

Using this formula we easily find that the summed series have the following form:

$$
G_{1}=(-1)^{-D}\left(K_{3}^{2}\right)^{D-6} \frac{\Gamma\left(\frac{D}{2}-2\right)^{3} \Gamma(D-5) \Gamma(5-D)}{\Gamma(D-3) \Gamma\left(\frac{3 D}{2}-6\right)},
$$

y:

$$
G_{5}=(-1)^{-D}\left(K_{3}^{2}\right)^{D-6} \frac{\Gamma\left(2-\frac{D}{2}\right) \Gamma\left(\frac{D}{2}-2\right) \Gamma\left(\frac{D}{2}-1\right) \Gamma(D-4) \Gamma(D-5) \Gamma(5-D)}{\Gamma(D-3) \Gamma\left(\frac{3 D}{2}-6\right)} .
$$

On the other hand we have that:

$$
G_{2}=(-1)^{-D}\left(K_{3}^{2}\right)^{D-6} \frac{\Gamma\left(3-\frac{D}{2}\right) \Gamma\left(\frac{D}{2}-2\right)^{2} \Gamma(6-D) \Gamma(D-5)^{2}}{\Gamma(D-3) \Gamma\left(\frac{3 D}{2}-6\right)},
$$

and :

$$
G_{3}=(-1)^{-D}\left(K_{3}^{2}\right)^{D-6} \frac{\Gamma\left(2-\frac{D}{2}\right) \Gamma\left(\frac{D}{2}-2\right) \Gamma\left(\frac{D}{2}-1\right) \Gamma(D-5) \Gamma\left(\frac{D}{2}-3\right) \Gamma\left(4-\frac{D}{2}\right) \Gamma(5-D)}{\Gamma(D-3) \Gamma\left(\frac{3 D}{2}-6\right) \Gamma\left(3-\frac{D}{2}\right)^{2}} .
$$


The hypergeometric function contained in the term $G_{7}$ and which has the form ${ }_{1} F_{0}$, can be transformed in another one of the type ${ }_{2} F_{1}$, and then we can apply the identity (42),

$$
{ }_{1} F_{0}\left(\begin{array}{c|c}
\alpha & 1
\end{array}\right)={ }_{2} F_{1}\left(\begin{array}{c|c}
\alpha, \beta & \beta
\end{array}\right),
$$

and then we get that

$$
G_{7}=0 .
$$

After a bit of algebra, and using known Gamma function properties, it is straightforward to show that the solutions (34) and (37) are equal in the limit $A=B=1$, giving

$$
\left(G_{1}+G_{5}\right)-\left(G_{2}+G_{3}\right)=0 .
$$

Therefore, defining $G_{I B F E}$ as the solution for the graph in (16), the final solution in general can be written as:

$$
G_{I B F E}=\left\{\begin{array}{l}
G_{2}+G_{3} \\
\text { or } \\
G_{1}+G_{5} .
\end{array}\right.
$$

\subsubsection{Validating the solution $G_{I B F E}$. Comparison with a conventional method : Integration by parts (IBP)}

When the propagator powers are integers, it is possible to evaluate such topologies with the integration by parts method [4. In the particular case of diagrams that contain subgraphs, this technique can be quite useful in order to simplify the integration problem, since it allows to simplify the geometry of the diagram.

In the particular case of the diagram which contain triangle subgraphs, this technique can be in order to simplify the integration problem. One very useful formula, which can be deduced applying IBP to a triangle graph, and which is known as the triangle identity, is the following:

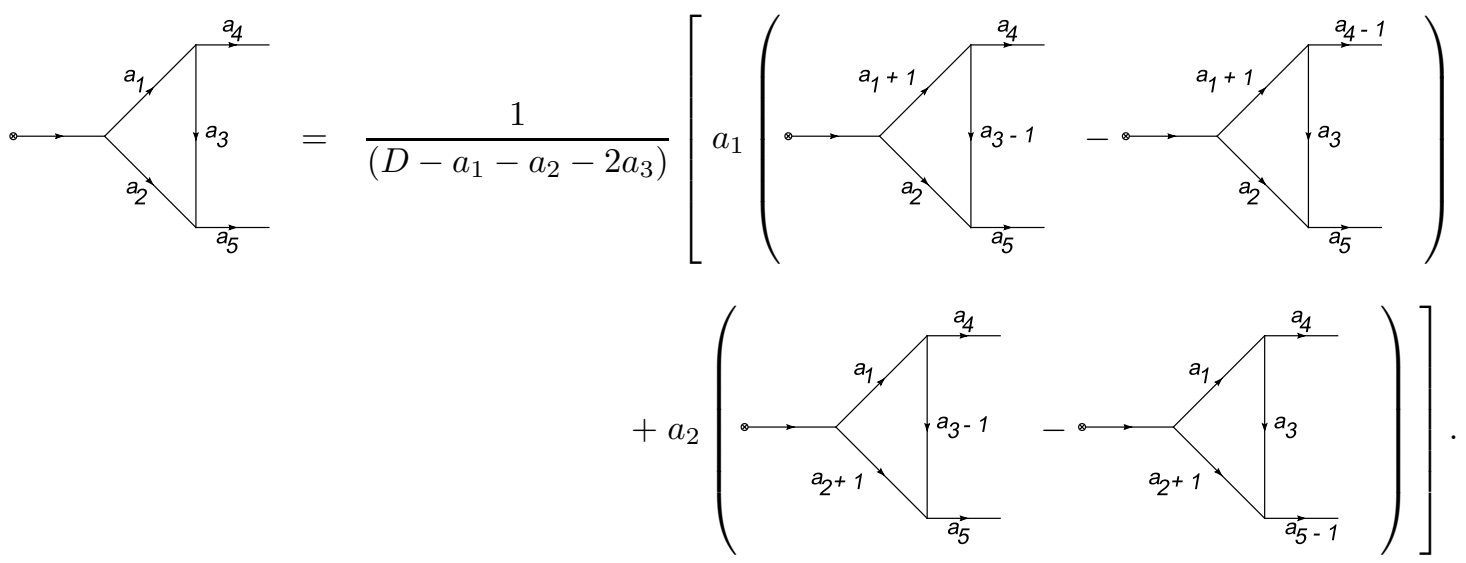

where it has been assumed that the indexes $a_{1}, a_{2}$, and $a_{3}$ have arbitrary values. We now apply directly this identity to the diagram depicted in (16), but taking all the propagator powers to be unity. The result that we obtain is: 

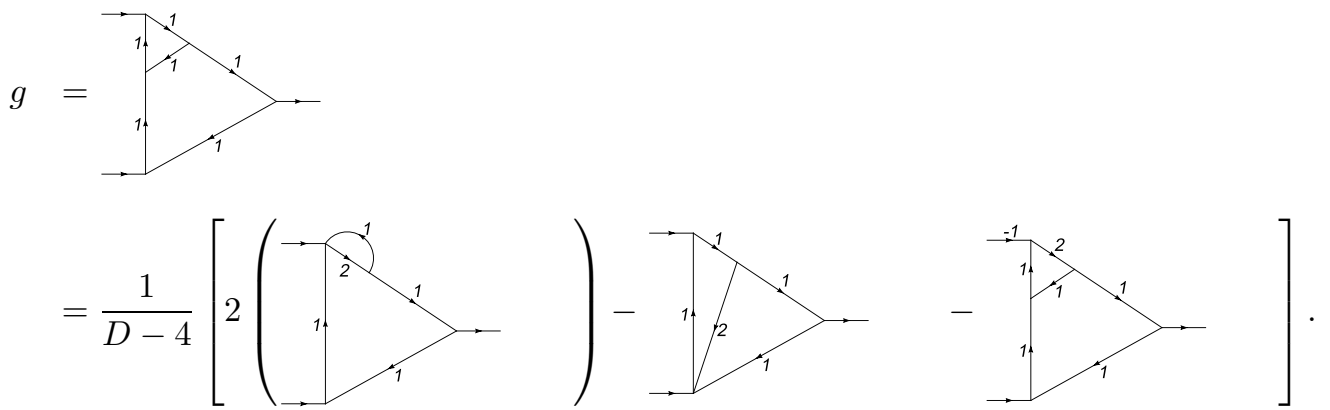

The third diagram in the left-hand side of the equation vanishes (external on-shell line condition). The second diagram can be further simplified using again (51), giving:

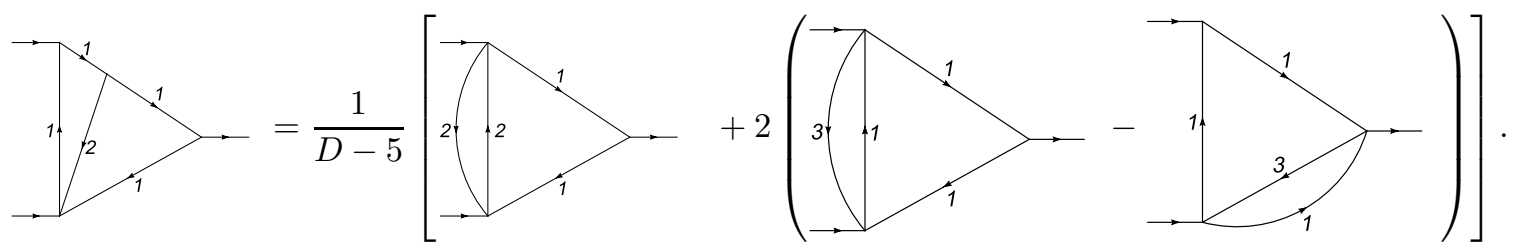

Now each term of the solution can be easily rewritten evaluating the bubble diagram and then the triangle. For this we need the general formulae for these graphs, given by:

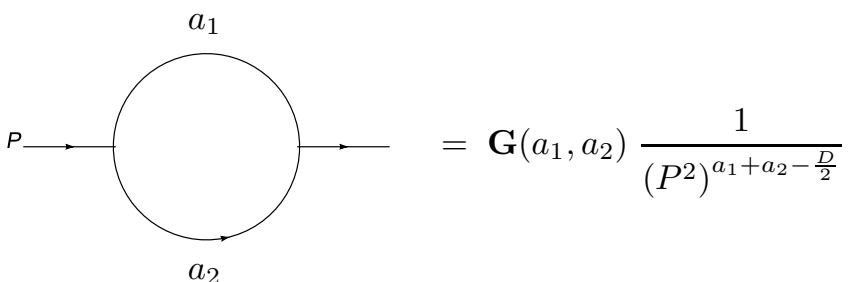

where:

$$
\mathbf{G}\left(a_{1}, a_{2}\right)=(-1)^{-D / 2} \frac{\Gamma\left(a_{1}+a_{2}-\frac{D}{2}\right) \Gamma\left(\frac{D}{2}-a_{1}\right) \Gamma\left(\frac{D}{2}-a_{2}\right)}{\Gamma\left(a_{1}\right) \Gamma\left(a_{2}\right) \Gamma\left(D-a_{1}-a_{2}\right)} .
$$

In the case of the triangle with conditions $P_{1}^{2}=P_{2}^{2}=0$ one has the fundamental formula:

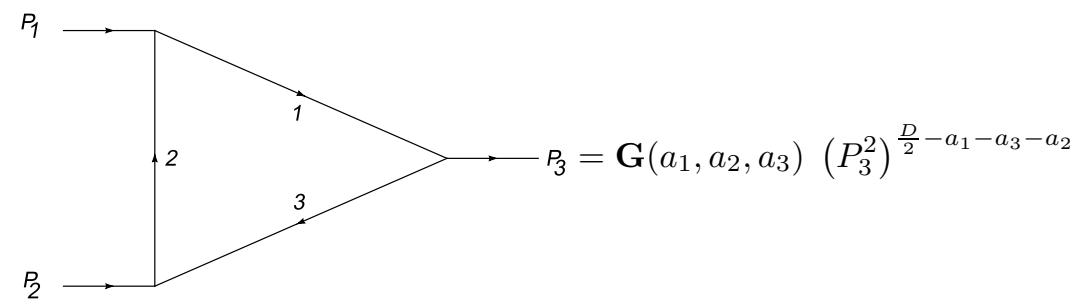

where the factor $\mathbf{G}\left(a_{1}, a_{2}, a_{3}\right)$ is given by:

$$
\mathbf{G}\left(a_{1}, a_{2}, a_{3}\right)=(-1)^{-D / 2} \frac{\Gamma\left(a_{1}+a_{2}+a_{3}-\frac{D}{2}\right) \Gamma\left(\frac{D}{2}-a_{2}-a_{3}\right) \Gamma\left(\frac{D}{2}-a_{1}-a_{2}\right)}{\Gamma\left(a_{1}\right) \Gamma\left(a_{3}\right) \Gamma\left(D-a_{1}-a_{3}-a_{2}\right)}
$$

Applying formulae (54) and (56), the solution for the diagram is then: 


$$
\begin{aligned}
g=G_{I B P}= & \frac{\left(K_{3}^{2}\right)^{D-6}}{D-4}\left[2 \mathbf{G}(1,2) \mathbf{G}\left(4-\frac{D}{2}, 1,1\right)-\frac{1}{D-5} \mathbf{G}(2,2) \mathbf{G}\left(1,4-\frac{D}{2}, 1\right)\right. \\
& \left.-\frac{1}{D-5} 2 \mathbf{G}(1,3)\left[\mathbf{G}\left(1,4-\frac{D}{2}, 1\right)-\mathbf{G}\left(1,1,4-\frac{D}{2}\right)\right]\right]
\end{aligned}
$$

Choosing one of the solutions for $G_{I B F E}($ (50), it can be easily shown that the solution of this diagram evaluated using the IBFE technique gives the same result as with IBP:

$$
G_{I B F E}=G_{I B P} .
$$

This is then a concrete confirmation of the IBFE technique, applied modularly to a diagram with triangle subgraphs. The important point is that this has allowed to obtain the MRE of the graph in a noticeably more direct and systematic way than finding it by taking Schwinger's parametric representation of the whole diagram.

\subsection{Example II : Two-loop propagator correction}

A more relevant example is the two-loop propagator correction, with five internal lines.

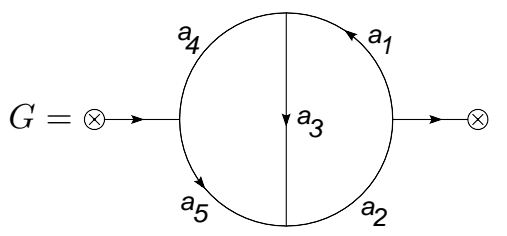

\subsubsection{Obtaining the diagram MRE}

In order to find the diagram MRE we apply formula (15) on the left-hand side of the diagram, obtaining the following graphical expression:

$$
G=\sum_{n_{1}, . ., n_{3}}\left(A_{1}\right)^{n_{1}}\left(A_{2}\right)^{n_{2}}\left(A_{3}\right)^{n_{3}} G_{T}\left(a_{1}, a_{2}, a_{3} ; n_{1}, n_{2}, n_{3}\right) \otimes \rightarrow-n_{-n_{3}} \otimes
$$

Using then the massless bubble one-loop functions $G_{A}$ [1], we quickly obtain the diagram MRE as:

$$
\begin{gathered}
G=\sum_{n_{1}, . ., n_{3}, l_{1}}\left(A_{1}\right)^{n_{1}}\left(A_{2}\right)^{n_{2}}\left(A_{3}\right)^{n_{3}} G_{T}\left(a_{1}, a_{2}, a_{3} ; n_{1}, n_{2}, n_{3}\right) \times G_{A}\left(a_{4}-n_{1}, a_{5}-n_{3} ; n_{7}\right) \\
\quad \stackrel{\left(-n_{2}-n_{7}\right)}{\longrightarrow} \otimes
\end{gathered}
$$

or equivalently:

$$
G=\sum_{n_{1}, . ., n_{3}, n_{7}}\left(A_{1}\right)^{n_{1}}\left(A_{2}\right)^{n_{2}}\left(A_{3}\right)^{n_{3}} G_{T}\left(a_{1}, a_{2}, a_{3} ; n_{1}, n_{2}, n_{3}\right) \times G_{A}\left(a_{4}-n_{1}, a_{5}-n_{3} ; n_{7}\right) \frac{1}{\left(p^{2}\right)^{-n_{2}-n_{7}}} .
$$

The one-loop functions $G_{T}$ and $G_{A}$ are given by the expressions:

$$
G_{T}\left(a_{1}, a_{2}, a_{3} ; n_{1}, n_{2}, n_{3}\right)=\frac{(-1)^{-D / 2}}{\prod_{j=1}^{3} \Gamma\left(a_{j}\right)} \sum_{n_{4}, . ., n_{6}} \phi_{n_{1}, . ., n_{6}} \frac{\prod_{j=1}^{4} \Delta_{j}}{\Gamma\left(\frac{D}{2}+n_{1}+n_{2}+n_{3}\right)},
$$


whose constraints are:

$$
\left\{\begin{aligned}
\Delta_{1} & =\left\langle\frac{D}{2}+n_{1}+n_{2}+n_{3}+n_{4}+n_{5}+n_{6}\right\rangle \\
\Delta_{2} & =\left\langle a_{1}+n_{1}+n_{2}+n_{4}\right\rangle \\
\Delta_{3} & =\left\langle a_{2}+n_{2}+n_{3}+n_{5}\right\rangle \\
\Delta_{4} & =\left\langle a_{3}+n_{1}+n_{3}+n_{6}\right\rangle
\end{aligned}\right.
$$

and for $G_{A}$ we have:

$$
G_{A}\left(a_{4}-n_{1}, a_{5}-n_{3} ; n_{7}\right)=\frac{(-1)^{-D / 2}}{\Gamma\left(a_{4}-n_{1}\right) \Gamma\left(a_{5}-n_{3}\right)} \sum_{n_{8}, n_{9}} \phi_{n_{7}, . ., n_{9}} \frac{\prod_{j=5}^{7} \Delta_{j}}{\Gamma\left(\frac{D}{2}+n_{7}\right)},
$$

where the constraints become:

$$
\left\{\begin{array}{l}
\Delta_{5}=\left\langle\frac{D}{2}+n_{7}+n_{8}+n_{9}\right\rangle \\
\Delta_{6}=\left\langle a_{4}-n_{1}+n_{7}+n_{8}\right\rangle \\
\Delta_{7}=\left\langle a_{5}-n_{3}+n_{7}+n_{9}\right\rangle
\end{array}\right.
$$

With this information we finally are able to write the diagram MRE as:

$$
G=\frac{(-1)^{-D}}{\prod_{j=1}^{3} \Gamma\left(a_{j}\right)} \sum_{n_{1}, . ., n_{9}} \phi_{n_{1}, . ., n_{9}} \frac{\left(A_{1}\right)^{n_{1}}\left(A_{2}\right)^{n_{2}}\left(A_{3}\right)^{n_{3}}\left(p^{2}\right)^{n_{2}+n_{7}}}{\Gamma\left(\frac{D}{2}+n_{1}+n_{2}+n_{3}\right) \Gamma\left(\frac{D}{2}+n_{7}\right)} \frac{\prod_{j=1}^{7} \Delta_{j}}{\Gamma\left(a_{4}-n_{1}\right) \Gamma\left(a_{5}-n_{3}\right)}
$$

This expansion contains $9 \Sigma$ and $7 \delta$, and therefore the solution of this diagram corresponds to a double series and since we are dealing with a propagator, the kinematical variable $\left(p^{2}\right)$ that is present does not appear explicitly as an argument in this series. We expect that for this type of diagrams the solution will not be a single term, since it does not correspond to an optimal topology (in the sense that the multiplicity of the resulting series does not depend on the number of loops or equivalently only depends on the invariants of the physical process [2]).

In order to simplify the notation in (68) we take $A_{1}=A, A_{2}=B$ and $A_{3}=C$. With these invariants six regions of interest can be generated, each one with a different solution, but in the limit $A=B=C=1$ they are all identical. These regions are given by:

$$
\left\{\begin{array}{l}
A<B<C, \\
A<C<B, \\
B<A<C, \\
B<C<A, \\
C<B<A, \\
C<A<B .
\end{array}\right.
$$

We arbitrarily take the region in which $(A<C<B)$. Then when we extract the different terms of the MRE (bivalued hypergeometric functions) we only sum those that have as one of their arguments the following simple combinations:

$$
\left(\frac{A}{C}\right),\left(\frac{A}{B}\right),\left(\frac{C}{B}\right)
$$

or equivalently all the double combinations generated when combining these simple combinations: $\left(\frac{A}{C}, \frac{C}{B}\right)$, $\left(\frac{A}{B}, \frac{C}{B}\right)$, etc. Given these conditions, the general solution that we get starting from (668) is the following:

$$
G=g_{1}+g_{2}+g_{3}+g_{4}+g_{5}+g_{6}
$$

where we have that: 


$$
\begin{aligned}
& g_{1}=(-1)^{-D}\left(p^{2}\right)^{D-a_{12345}} \frac{\Gamma\left(a_{13}-\frac{D}{2}\right) \Gamma\left(\frac{D}{2}-a_{23}\right) \Gamma\left(\frac{D}{2}-a_{1}\right) \Gamma\left(a_{1345}-D\right) \Gamma\left(\frac{D}{2}-a_{5}\right) \Gamma\left(D-a_{134}\right)}{\Gamma\left(a_{1}\right) \Gamma\left(a_{3}\right) \Gamma\left(a_{5}\right) \Gamma\left(D-a_{123}\right) \Gamma\left(a_{134}-\frac{D}{2}\right) \Gamma\left(\frac{3 D}{2}-a_{1345}\right)} \\
& \times A^{\frac{D}{2}-a_{13}} B^{-a_{2}} \\
& \times F^{2: 1: 1: 1}\left(\begin{array}{cc}
\left\{a_{2}, \frac{D}{2}-a_{1}\right\}, & \left\{1-a_{5}, \frac{D}{2}-a_{5}\right\}, \\
\left\{\frac{3 D}{2}-a_{1345}, D+1-a_{1345}\right\}, & \left\{1-\frac{D}{2}+a_{23}\right\},
\end{array}\right.
\end{aligned}
$$

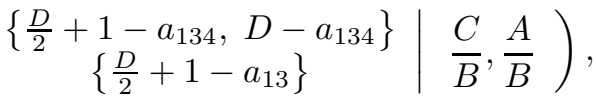

$$
\begin{aligned}
& g_{2}=(-1)^{-D}\left(p^{2}\right)^{D-a_{12345}} \frac{\Gamma\left(\frac{D}{2}-a_{45}\right) \Gamma\left(D-a_{2345}\right) \Gamma\left(a_{12345}-D\right) \Gamma\left(\frac{D}{2}-a_{13}\right) \Gamma\left(a_{345}-\frac{D}{2}\right)}{\Gamma\left(a_{1}\right) \Gamma\left(a_{2}\right) \Gamma\left(a_{3}\right) \Gamma\left(\frac{D}{2}\right) \Gamma\left(D-a_{123}\right)} \\
& \times B^{D-a_{12345}} C^{a_{45}-\frac{D}{2}}
\end{aligned}
$$

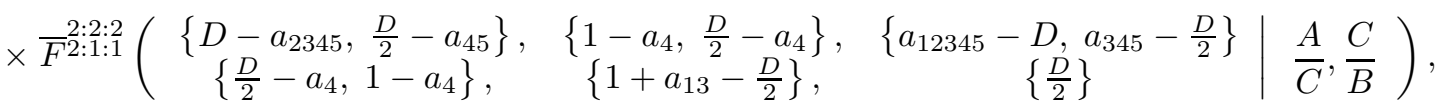

$$
\begin{aligned}
& g_{3}=(-1)^{-D}\left(p^{2}\right)^{D-a_{12345}} \frac{\Gamma\left(a_{13}-\frac{D}{2}\right) \Gamma\left(a_{12345}-D\right) \Gamma\left(D-a_{1345}\right) \Gamma\left(\frac{3 D}{2}-2 a_{3}-a_{1245}\right) \Gamma\left(a_{345}-\frac{D}{2}\right)}{\Gamma\left(a_{1}\right) \Gamma\left(a_{2}\right) \Gamma\left(a_{3}\right) \Gamma\left(\frac{D}{2}\right) \Gamma\left(D-a_{123}\right)} \\
& \times A^{\frac{D}{2}-a_{13}} B^{D-a_{12345}} C^{a_{1345}-\frac{D}{2}} \\
& \times \bar{F}^{2: 1: 1: 1}\left(\begin{array}{cc}
\left\{D-a_{1345}, \frac{3 D}{2}-2 a_{3}-a_{1245}\right\}, & \left\{\frac{D}{2}+1-a_{134}, D-a_{134}\right\}, \\
\left\{D-a_{134}, \frac{D}{2}+1-a_{134}\right\}, & \left\{\frac{D}{2}+1-a_{13}\right\},
\end{array}\right.
\end{aligned}
$$

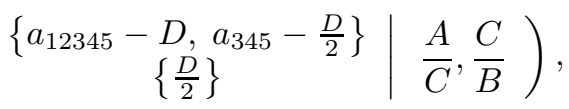

$$
\begin{aligned}
g_{4}= & (-1)^{-D}\left(p^{2}\right)^{D-a_{12345}} \frac{\Gamma\left(\frac{D}{2}-a_{13}\right) \Gamma\left(\frac{D}{2}-a_{23}\right) \Gamma\left(a_{123}-\frac{D}{2}\right) \Gamma\left(a_{45}-\frac{D}{2}\right) \Gamma\left(\frac{D}{2}-a_{5}\right) \Gamma\left(\frac{D}{2}-a_{4}\right)}{\Gamma\left(a_{1}\right) \Gamma\left(a_{2}\right) \Gamma\left(a_{5}\right) \Gamma\left(D-a_{45}\right) \Gamma\left(D-a_{123}\right)} \\
& \times A^{\frac{D}{2}-a_{13}} B^{D-a_{12345}} C^{a_{1345}-\frac{D}{2}} \\
& \times F^{2: 2: 1: 1}\left(\begin{array}{ccc}
\left\{a_{3}, a_{123}-\frac{D}{2}\right\}, & \left\{1-a_{4}, \frac{D}{2}-a_{4}\right\}, & \left\{1-a_{5}, \frac{D}{2}-a_{5}\right\} \\
\left\{D-a_{45}, 1+\frac{D}{2}-a_{45}\right\}, & \left\{1-\frac{D}{2}+a_{13}\right\}, & \left\{1-\frac{D}{2}+a_{23}\right\}
\end{array} \mid \frac{A}{B}, \frac{C}{B}\right),
\end{aligned}
$$




$$
\begin{aligned}
& g_{5}=(-1)^{-D}\left(p^{2}\right)^{D-a_{12345}} \frac{\Gamma\left(a_{13}-\frac{D}{2}\right) \Gamma\left(a_{23}-\frac{D}{2}\right) \Gamma\left(\frac{D}{2}-a_{3}\right) \Gamma\left(D-a_{134}\right) \Gamma\left(D-a_{235}\right)}{\Gamma\left(a_{1}\right) \Gamma\left(a_{2}\right) \Gamma\left(a_{3}\right) \Gamma\left(2 D-a_{1245}-2 a_{3}\right) \Gamma\left(a_{134}-\frac{D}{2}\right)} \\
& \times \frac{\Gamma\left(a_{1245}+2 a_{3}-\frac{3 D}{2}\right)}{\Gamma\left(a_{235}-\frac{D}{2}\right)} A^{\frac{D}{2}-a_{13}} B^{a_{3}-\frac{D}{2}} C^{\frac{D}{2}-a_{23}} \\
& \times F^{2: 1: 1: 1}\left(\begin{array}{cc}
\left\{D-a_{123}, \frac{D}{2}-a_{3}\right\}, & \left\{\frac{D}{2}+1-a_{235}, D-a_{235}\right\}, \\
\left\{2 D-a_{1245}-2 a_{3}, \frac{3 D}{2}+1-2 a_{3}-a_{1245}\right\}, & \left\{\frac{D}{2}+1-a_{23}\right\},
\end{array}\right.
\end{aligned}
$$

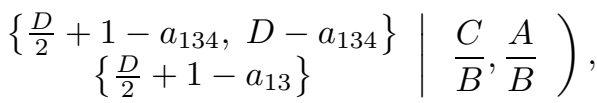

and finally:

$$
\begin{aligned}
& g_{6}=(-1)^{-D} \frac{\Gamma\left(\frac{D}{2}-a_{13}\right) \Gamma\left(a_{23}-\frac{D}{2}\right) \Gamma\left(\frac{D}{2}-a_{2}\right) \Gamma\left(\frac{D}{2}-a_{4}\right) \Gamma\left(a_{2345}-D\right) \Gamma\left(D-a_{235}\right)}{\Gamma\left(a_{2}\right) \Gamma\left(a_{3}\right) \Gamma\left(a_{4}\right) \Gamma\left(D-a_{123}\right) \Gamma\left(a_{235}-\frac{D}{2}\right) \Gamma\left(\frac{3 D}{2}-a_{2345}\right)} \\
& \times B^{-a_{1}} C^{\frac{D}{2}-a_{23}} \\
& \times F^{2: 1: 2: 1}\left(\begin{array}{cc}
\left\{a_{1}, \frac{D}{2}-a_{2}\right\}, & \left\{1-a_{4}, \frac{D}{2}-a_{4}\right\}, \\
\left\{\frac{3 D}{2}-a_{2345}, 1+D-a_{2345}\right\}, & \left\{1-\frac{D}{2}+a_{13}\right\},
\end{array}\right.
\end{aligned}
$$

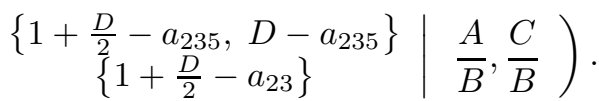

The previous results have been written in terms of the functions $F$ and $\bar{F}$ (for more information see Ref. 2] ), which are defined as:

$$
\bar{F}^{p: r: s: v}\left(\begin{array}{ccc}
\left\{\gamma_{1}, . ., \gamma_{p}\right\} & \left\{a_{1}, . ., a_{r}\right\} & \left\{c_{1}, . ., c_{u}\right\} \\
\left\{\beta_{1}, . ., \beta_{q}\right\} & \left\{b_{1}, . ., b_{s}\right\} & \left\{d_{1}, . ., d_{v}\right\}
\end{array} \mid x, y\right)=\sum_{n, m}^{\infty} \frac{\prod_{j=1}^{p}\left(\gamma_{j}\right)_{n-m} \prod_{j=1}^{r}\left(a_{j}\right)_{n} \prod_{j=1}^{u}\left(c_{j}\right)_{m}}{\prod_{j=1}^{q}\left(\beta_{j}\right)_{n-m} \prod_{j=1}^{s}\left(b_{j}\right)_{n} \prod_{j=1}^{v}\left(d_{j}\right)_{m}} \frac{x^{n}}{n !} \frac{y^{m}}{m !},
$$

and similarly we also have:

$$
F \text { F:r:s:u }\left(\begin{array}{ccc}
\left\{\gamma_{1}, . ., \gamma_{p}\right\} & \left\{a_{1}, . ., a_{r}\right\} & \left\{c_{1}, . ., c_{u}\right\} \\
\left\{\beta_{1}, . ., \beta_{q}\right\} & \left\{b_{1}, . ., b_{s}\right\} & \left\{d_{1}, . ., d_{v}\right\}
\end{array} \mid x, y\right)=\sum_{n, m}^{\infty} \frac{\prod_{j=1}^{p}\left(\gamma_{j}\right)_{n+m} \prod_{j=1}^{r}\left(a_{j}\right)_{n} \prod_{j=1}^{u}\left(c_{j}\right)_{m}}{\prod_{j=1}^{q}\left(\beta_{j}\right)_{n+m} \prod_{j=1}^{s}\left(b_{j}\right)_{n} \prod_{j=1}^{v}\left(d_{j}\right)_{m}} \frac{x^{n}}{n !} \frac{y^{m}}{m !} .
$$

This last series is called Kampé de Fériet function. Finally the solution is obtained by simply taking $A=B=$ $C=1$.

We have solved this diagram in a modular or loop by loop form. In order to appreciate the advantages of this way of applying IBFE, it is important to compare the MRE (68) with the MRE of the same diagram obtained by the application of IBFE to the parametric integral of the complete diagram. For this purpose let us consider first Schwinger's parametric representation of the complete diagram, which is given by:

$$
G=\frac{(-1)^{-D}}{\prod_{j=1}^{5} \Gamma\left(a_{j}\right)} \int_{0}^{\infty} d \vec{x} \frac{\exp \left[-\frac{F}{\left(x_{1}+x_{2}\right)\left(x_{3}+x_{4}+x_{5}\right)+x_{3}\left(x_{4}+x_{5}\right)}\right]}{\left[\left(x_{1}+x_{2}\right)\left(x_{3}+x_{4}+x_{5}\right)+x_{3}\left(x_{4}+x_{5}\right)\right]^{\frac{D}{2}}},
$$


where the polynomial $F$ is given by:

$$
F=\left[x_{1} x_{2}\left(x_{3}+x_{4}+x_{5}\right)+x_{4} x_{5}\left(x_{1}+x_{2}\right)+x_{3} x_{4} x_{5}+x_{1} x_{3} x_{5}+x_{2} x_{3} x_{4}\right] p^{2} .
$$

If we now obtain the diagram MRE using (79) we can compare (Table I) this MRE with MRE (68):

\begin{tabular}{lcc}
\hline & IBFE complete diagram & IBFE modular \\
\hline Multiplicity multiregion series $(\sigma)$ & 12 & 9 \\
Kronecker deltas of the expansion $(\delta)$ & 8 & 7 \\
Multiplicity resulting series $(\sigma-\delta)$ & 4 & 2 \\
Possible contributions to the solutions $\left(C_{\delta}^{\sigma}\right)$ & 495 & 36 \\
\hline
\end{tabular}

(Table I)

We find that the MRE obtained in terms of the one-loop functions $G_{T}$ is considerably reduced with respect to the one that we get when IBFE is applied to both loops of the diagram simultaneously, not only in the number of sums and Kronecker deltas, but also the difference between them is reduced, which implies that the multiplicity of the resulting series is less, a nontrivial fact that simplifies considerably the complexity of the solutions.

\section{Commentaries}

The calculation technology that we have presented here can be useful in order to find minimal expressions for the MRE of diagrams that contain triangular subgraphs, such as those that we see in the following figure:
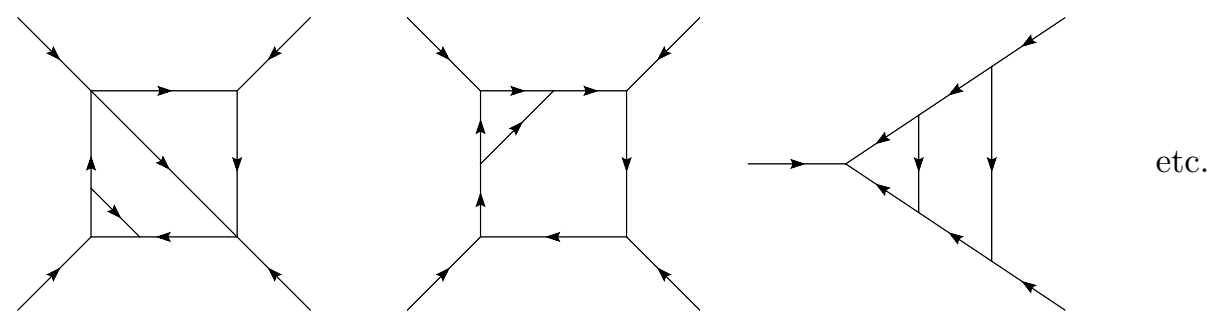

The loop function $G_{T}$ is very useful since it opens up the possibility of evaluating other families of graphs and to obtain solutions with less complexity and less number of terms when compared with the application of IBFE to a parametric integral of the complete diagram. Although we have presented here the one-loop function $G_{T}$ for the massless case, it is certainly possible to consider also massive propagators and define new loop functions for these cases. It will be always possible to use here the same reduction procedure for diagrams that contain bubbles or triangles that we have used for the massless cases.

The same idea can be extended to consider one-loop functions for modules that contain four or more propagators. Nevertheless, the reductions are no longer so simple as for the bubble and triangle subgraphs. The important point is that the modular IBFE application, particularly when it is done loop by loop, allows to obtain a minimal representation for the diagram MRE, from which the analytical solution follows.

\section{Conclusions}

The general solutions that can be obtained in the evaluation of Feynman diagrams always correspond to multivariable hypergeometric series, whose multiplicity can be deduced directly from the MRE that represents the diagram. One of the characteristics of the IBFE technique is that it has a lower bound that fixes the multiplicity $\mu$ of these hypergeometric series, and which is related to the minimal number of invariants $(N)$ that characterizes a specific Feynman diagram, described through the formula:

$$
\mu \geqslant(N-1)
$$


The greater-equal sign is here included since besides the number of invariants one must consider also the topological family to which the diagram belongs. If this is not the optimal for IBFE application, in the sense described in [2, then the multiplicity is also increased according to the number of loops in the diagram. This multiplicity can be directly recognized from the MRE of the diagram, and it is equal to the difference between the number of sums and constraints (Kronecker deltas) that are present. Solutions with 0 multiplicity (which in reality is not a series, and contains only one term), or multiplicity 1 or 2 , correspond to series whose properties are extensively discussed in the literature [5, 6, 7, 8]. Nevertheless, for triple or higher multiplicity series, the information is rather limited and it is therefore important to have methods that allow to reduce the diagram MRE, not only in the sense of lowering the number of sums and deltas, but also to reduce their difference, which is something that we have shown here can be done modularly (see Example II).

The idea of the modular application of IBFE can be easily extended to one-loop modules or subgraphs that contain four or more propagators. The result will be in general a reduced MRE, that will also contain simpler solution, i.e. hypergeometric functions of lower multiplicity. In the same way, the idea of using fictitious invariants for selecting the correct solution associated to a diagram can be also generalized to all the cases in which hypergeometric functions with unit arguments are generated.

The modular application of the integration technique IBFE has shown to be a powerful calculational tool, not only for its simplicity but also because it allows to calculate many families of graphs. Here we have seen that the systematical graphical procedure that we have implemented for triangular modules, makes the reduction just as simple as in the case of bubbles contained in a diagram.

\section{Acknowledgements :}

We acknowledge support from Fondecyt under Grant No. 3080029.

\section{References}

[1] I.Gonzalez, I.Schmidt, Phys. Rev. D78 (2008) 086003.

[2] I.Gonzalez, I.Schmidt, Nucl. Phys. B 769 (2007) 124-173.

[3] I.Gonzalez, I.Schmidt, Phys. Rev. D72 (2005) 106006.

[4] F.V. Tkachov, Phys. Lett. B100, 65 (1981); K.G. Chetyrkin and F.V. Tkachov, Nucl. Phys. B192 (1981) 159.

[5] W.N.Bailey, Generalized Hypergeometric Functions (Stechert-Hafner Service Agency, New York and London, 1964).

[6] L.J.Slater, Generalized Hypergeometric Functions (Cambridge University Press, Cambridge, England, 1966).

[7] L.S.Gradshteyn, L.M.Ryzhik, Table of Integrals, Series, and Products (Academic Press, New York, 2000), 6th ed.

[8] H. Exton, Multiple Hypergeometric Functions and Applications (Ellis Horwood,Westergate, England,1976). 